\title{
Importance of Central Aortic Pressure
}

\author{
Shantaram V. ${ }^{1}$ \\ ${ }^{1}$ Department of General Medicine, Nizam's Institute of Medical \\ Sciences, Hyderabad, Telangana, India
}

Indian J Cardiovasc Dis Women-WINCARS 2017;2:65-66

Hypertension is a dangerous condition in which blood pressure (BP) is persistently higher than normal. If left untreated, people with hypertension are at risk for heart attack, heart failure, stroke, and kidney failure.

However, systolic pressure varies throughout the arterial tree, such that aortic (central) systolic pressure is actually lower than corresponding brachial values, although this difference is highly variable between individuals. From a physiologic viewpoint, central aortic blood pressure (CABP), at least in elderly, is likely to be more relevant than the peripheral BP. CABP has a stronger correlation with the degree of left ventricular hypotrophy, heart failure, myocardial infarction (MI), and stroke than cuff pressure recording. ${ }^{1}$

Owing to vascular aging, arterial distensibility decreases with age, and this leads to increased systolic pressure in the aorta and increased pulse pressure consistently. Several limitations exist with peripheral blood pressure. First, central aortic pressure is a better predictor of cardiovascular outcome than peripheral pressure. Second, peripherally obtained BP does not accurately reflect central pressure because of pressure amplification. Last, antihypertensive medications have differing effects on central pressures despite similar reductions in brachial BP. Therefore, it is better to consider the CABP for therapeutic interventions. Even though the most accurate way of recording CABP is direct pressure recording from the catheter in the aorta during cardiac catheterization, the most practical and easiest way is to derive CABP by noninvasive methods such as applanation tonometry. ${ }^{2}$

Certain drugs that are generally used, such as $\beta$-blockers, may not lower the central BP. The Conduit Artery Function Evaluation (CAFE) study, a substudy of the Anglo Scandinavian Cardiac Outcomes Trial (ASCOT), examined the impact of two different BP-lowering regimens (atenolol \pm thiazide-based vs. amlodipine \pm perindopril-based therapy) on derived central aortic pressures and hemodynamics. ${ }^{3}$ Metaanalysis showed that atenolol is effective at decreasing the cuff BP but not CABP. Drugs of choice would be calcium channel blocker and angiotensin II receptor blocker (ARB) in elderly people along with diuretics such as chlorthalidone. They have greater therapeutic safety in their property of lowering central BP in elderly people. ${ }^{4}$

DOI https://doi.org/ 10.1055/s-0038-1622967.
Address for correspondence Shantaram V., MD, House No: 401, Satya Sai Prema Kuteer, Srinagar Colony, Hyderabad, TS 500073, India (e-mail: phanivvsoffice@gmail.com).

The PEAR (Pharmacogenomic Evaluation of Antihypertensive Responses) study was done to identify demographic and clinical factors associated with central pressures in patients with uncomplicated hypertension. Significant predictors of central systolic blood pressure (SBP) included height, smoking status, heart rate (HR), and peripheral SBP, whereas central diastolic blood pressure (DBP) was explained by peripheral DBP and HR. ${ }^{5}$ Various studies have shown that up to 80 years of age, SBP of 140 has to be the cutoff point. In very elderly people older than 80 years, probably 150 and other levels could be individualized. ${ }^{6}$

In the present issue of the journal, Hemamalini et al presented the data of the CABP from the south Indian population, as there are limited studies from this population. They found that CABP is statistically significantly lower than the manually recorded peripheral SBP irrespective of sex, hypertension, diabetes status, and use of $\beta$-blockers. A small but significant difference was observed between machinederived peripheral DBP and central aortic DBP.

\section{References}

1 McEniery CM, Cockcroft JR, Roman MJ, Franklin SS, Wilkinson IB. Central blood pressure: current evidence and clinical importance. Eur Heart J 2014;35(26):1719-1725

2 Nelson MR, Stepanek J, Cevette M, Covalciuc M, Hurst RT, Tajik AJ. Noninvasive measurement of central vascular pressures with arterial tonometry: clinical revival of the pulse pressure waveform? Mayo Clin Proc 2010;85(5):460-472

3 Williams B, Lacy PS, Thom SM, et al; CAFE Investigators; Anglo-Scandinavian Cardiac Outcomes Trial Investigators; CAFE Steering Committee and Writing Committee. Differential impact of blood pressure-lowering drugs on central aortic pressure and clinical outcomes: principal results of the Conduit Artery Function Evaluation (CAFE) study. Circulation 2006;113(9):1213-1225

4 Epstein BJ, Anderson S. Discordant effects of beta-blockade on central aortic systolic and brachial systolic blood pressure: considerations beyond the cuff. Pharmacotherapy 2007;27(9):1322-1333

5 Rosenwasser RF, Shah NK, Smith SM, et al. Baseline predictors of central aortic blood pressure: a PEAR substudy. J Am Soc Hypertens 2014;8(3):152-158

6 Denker MG, Cohen DL. What is an appropriate blood pressure goal for the elderly: review of recent studies and practical recommendations. Clin Interv Aging 2013;8:1505-1517
License terms

(®) $\Theta \circledast$ 